\title{
Cross talks between storage ring magnets at the Extremely Brilliant Source at the European Synchrotron Radiation Facility
}

\author{
Gaël Le Bec®, Joël Chavanne, Simone Liuzzo®, and Simon White® \\ ESRF-The European Synchrotron, CS40220, 38043 Grenoble Cedex 9, France
}

(Received 16 March 2021; accepted 16 June 2021; published 28 July 2021)

\begin{abstract}
Significant magnetic cross talks were observed between neighboring magnets on the Extremely Brilliant Source (EBS) low emittance storage ring. The main sources for these cross talks are the short longitudinal distances between magnets, which reach $47 \mathrm{~mm}$ in some cases. It affects mainly the dipole bending angles and the quadrupole gradients. A $1 \%$ bending angle correction was applied to the (permanent) bending magnets before their installation in the storage ring to compensate for the cross talks, while gradient errors as high as $1.8 \%$ were observed on quadrupoles. Intensive 3D magnetic simulations gave the longitudinal distribution of the cross talk errors for various magnet pairs and current settings. The error distribution depends on the magnet pairs: cross talks may create errors at magnet edges or all along the magnets, with same or opposite polarity. The simulations were validated by magnetic measurements, the discrepancies between measured and simulated values being in the $10^{-4}$ range with respect to the nominal gradients. Cross talk induced gradient errors were added to all quadrupoles and neighboring magnets in the lattice model. Even if it complicated the commissioning, the cross talks have no impact on the final performances of the lattice as the design machine parameters were reached for the most part.
\end{abstract}

DOI: 10.1103/PhysRevAccelBeams.24.072401

\section{INTRODUCTION}

The Extremely Brilliant Source (EBS) is an upgrade of the European Synchrotron Radiation Facility (ESRF) light source. It relies on a new low emittance storage ring based on the Hybrid Multibend Achromat lattice (HMBA) [1], derived from the Multibend Achromat lattice implemented at MAX IV in Sweden [2]. The facility started to deliver photons for users in August 2020, eight years after the beginning of the project. Most of the design parameters have been reached, e.g., $135 \mathrm{pm}$ rad horizontal emittance, 21 hours lifetime and $200 \mathrm{~mA}$ beam current. As a new type of storage ring, it inspired several light source upgrade projects around the world [3-6], some of them being in the construction phase.

All of these storage rings are characterized by an increased number of bending magnets (the ESRF-EBS counts seven dipoles per arc) and by strong quadrupoles with gradients ranging from 50 to $100 \mathrm{~T} / \mathrm{m}$. On top of that, most of the light source upgrade projects have to fit existing buildings. These constraints demand that the storage ring lattices be compact in the longitudinal direction.

\footnotetext{
*lebec@esrf.f
}

Published by the American Physical Society under the terms of the Creative Commons Attribution 4.0 International license. Further distribution of this work must maintain attribution to the author(s) and the published article's title, journal citation, and DOI.
The free space between the ESRF-EBS magnets is shorter than in most machines. The distance between coils of adjacent magnets is less than one centimeter in most cases and the pole-to-pole distance is as small as $47 \mathrm{~mm}$ in the worst case (Fig. 1). Table I gives the pole-to-pole distance between adjacent magnets and Table II gives the magnet apertures. One can note that the ratio of the distance between adjacent magnets and the sum of their apertures is

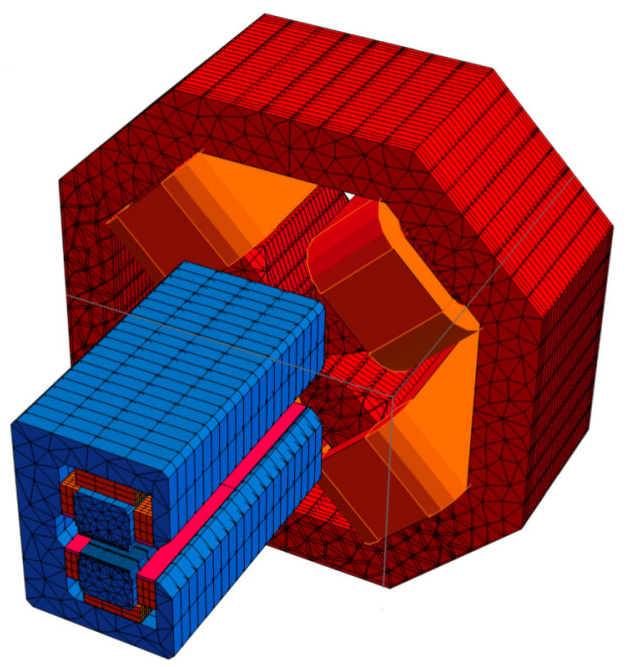

FIG. 1. View of a 3D magnetic model of interacting moderate gradient quadrupole (in red) and permanent magnet dipole module (in blue). The DL dipoles are built with five modules with different field values. 
TABLE I. Longitudinal distances between magnet poles (distances greater than $150 \mathrm{~mm}$ are not reported). Magnets QF1, QD2, QD3, QF4 and QD5 are moderate gradient quadrupoles, QF6 and QF8 are high gradient quadrupoles, SD and SF are sextupoles, OD are octupoles, DQ are combined dipole-quadrupoles and DL are permanent magnet dipoles. The last column indicates intensity of the cross talk. It is high for most of the moderate gradient quadrupoles and their neighbors.

\begin{tabular}{llcl}
\hline \hline Magnet 1 & \multicolumn{1}{c}{ Magnet 2 } & Distance $(\mathrm{mm})$ & Cross talk \\
\hline QF1 & Corrector & 110 & Low \\
QD2 & DL1 (high field) & 47 & High \\
QD3 & DL1 (low field) & 47 & High \\
QD3 & SD1 & 75 & High \\
QF4 & SF2 & 75 & High \\
QF4 & OD & 60 & Highest \\
QD5 & SD1 & 75 & High \\
QF6 & DL2 (high field) & 150 & Low \\
QF6 & DQ1 & 90 & Low \\
QF8 & DQ1 & 90 & Low \\
DQ2 & Corrector & 93 & Low \\
\hline \hline
\end{tabular}

TABLE II. Magnet apertures: central gaps for dipoles and bore diameter for higher order multipoles. The QF1-QD5 moderate gradient quadrupoles have 2.5 to $2.9 \mathrm{~mm}$ pole chamfers. All other magnets have straight poles.

\begin{tabular}{llcc}
\hline \hline Magnet name & \multicolumn{1}{c}{ Type } & Aperture & \\
\hline DL1-DL2 & Dipoles (high field side) & 25.5 & $\mathrm{~mm}$ \\
DL1-DL2 & Dipoles (low field side) & 30.5 & $\mathrm{~mm}$ \\
QF1-QD5 & Moderate gradient quadrupole & 32.8 & $\mathrm{~mm}$ \\
QF6-QF8 & High gradient quadrupole & 25.4 & $\mathrm{~mm}$ \\
SD1-SF2 & Sextupoles & 38.4 & $\mathrm{~mm}$ \\
OD & Octupoles & 37.2 & $\mathrm{~mm}$ \\
SH & Correctors & 33 & $\mathrm{~mm}$ \\
\hline \hline
\end{tabular}

close to one in all the cases where strong cross talks were observed. Figure 2 shows one standard cell with its 34 magnets. The compactness of the machine is certainly the main source of magnetic cross talks.

Most light source upgrade projects may suffer from cross talks due to their longitudinal compactness. The cross talk between a dipole and a quadrupole was studied in simulation in the context of the Advanced Photon Source (APS) upgrade [8].

The magnetic saturation is another contributor to cross talks. Due to their high gradients and dc operation, the ESRF-EBS storage ring quadrupoles were designed to work in the saturation region. Magnet designers often use the saturation ratio as a global indicator of the magnetic saturation in magnets. This ratio is defined as $S=\left(B_{\mathrm{LIN}}-B_{\mathrm{SAT}}\right) / B_{\mathrm{LIN}}$, where the subscripts indicate whether the field values are linearly extrapolated from low current values $\left(B_{\mathrm{LIN}}\right)$ or real, saturated values $\left(B_{\mathrm{SAT}}\right)$. We emphasize the fact that this indicator is not a design

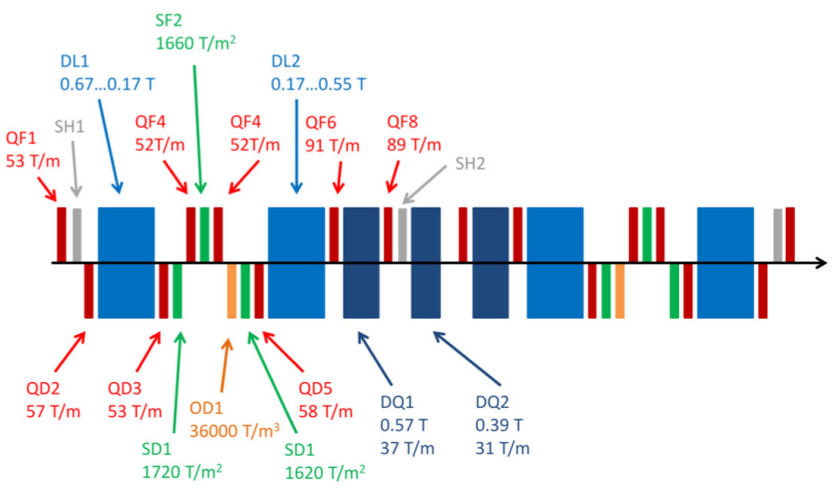

FIG. 2. Simplified view of the lattice magnets of a standard cell of the ESRF-EBS [7]. QD and QF are quadrupoles, DQ are dipole-quadrupoles, DL are dipoles, SD and SF are sextupoles, $\mathrm{OD}$ are octupoles, and SH are (sextupole-like) dipole and skew quadrupole correctors.

quality criterion, as saturated magnets can be low power and have a homogeneous field.

The saturation levels are shown in Fig. 3. Moderate gradient quadrupoles, with gradients close to $50 \mathrm{~T} / \mathrm{m}$, have a saturation ratio close to $5 \%$ which is relatively high but not unusual. The saturation level of the high gradient quadrupoles is as high as $17 \%$, but we will show that these quadrupoles are less affected by magnetic cross talk due to the increased longitudinal distances between magnets at their locations.

Among the seven dipoles installed in each cell, four are permanent magnets not equipped with any trim coils [9].

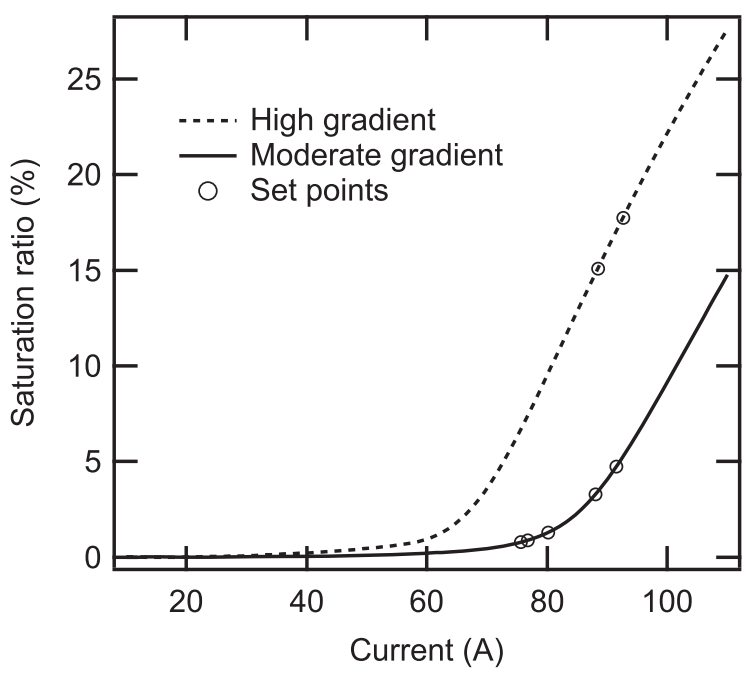

FIG. 3. Magnetic saturation ratio $S$ for the ESRF-EBS quadrupoles. The circles indicate the operation settings. The maximum field gradients are $52.8 \mathrm{~T} / \mathrm{m}$ at $91.5 \mathrm{~A}$ for the moderate gradient magnets and $91.1 \mathrm{~T} / \mathrm{m}$ at $92 \mathrm{~A}$ for high gradient magnets. The moderate gradient quadrupoles have a $32.8 \mathrm{~mm}$ bore diameter and are built with 1400-100 iron-silicon laminations, while the high gradient quadrupoles have a $25.4 \mathrm{~mm}$ bore diameter and are made of bulk AISI-1006 low carbon steel parts [7]. 
When these dipoles were finely tuned in the laboratory with stretched-wire measurement benches (Sec. II B), it was necessary to evaluate the influence of the neighboring quadrupoles on the bending angle. The cross talk was compensated by slightly increasing the bending angle during the tuning in the laboratory, in order to obtain the nominal integrated field once the magnet is installed on the girders.

The effect of magnetic cross talks on the integrated gradients was considered at the early stages of the project. It was studied on prototypes, but at that time the agreement between simulations and measurements was not good enough. The presence of permanent dipoles in the machine drew attention to the bending angles, making the gradient errors less visible: even if their orders of magnitude were known before the commissioning, they were not integrated in the magnet calibrations.

Two magnetic measurement datasets were available for each magnet: measurements done at the magnet supplier's premises with stretched-wire benches provided by the ESRF, and measurements done with similar benches on the ESRF site. These two sets helped us to identify a few wrong measurements at the supplier's premises or at the ESRF, mainly due to wrong magnet cyclings or wrong current settings. After correction of these errors, the relative uncertainty about the quadrupole calibrations was estimated at $U=3.2 \times 10^{-4}$ (this includes not only the benches but the power supplies and current readings). These small errors were not compatible with the large discrepancies observed between lattice model and optics measurements. The magnetic cross talks then appeared as large contributors to systematic calibration errors.

The next section of this paper presents the 3D simulation tools used to compute hundreds of pairs of magnet models at various currents. It also describes the measurement methods employed to validate the simulations. Section III presents the magnetic cross talks in the main cases: dipoles to quadrupoles, sextupoles to quadrupoles and octupoles to quadrupoles. The results, and the impact of cross talks on the ESRF-EBS performance, are summarized in the last section.

\section{SIMULATION AND MEASUREMENT METHODS}

\section{A. Magnetostatic simulations}

The simulation results presented in this paper were obtained with the RADIA code [10,11]. RADIA has the peculiarity of relying on a boundary integral method rather than on a finite element method (FEM). This was beneficial for the present study, as it allowed to easily separate the contributions from different sources.

A RADIA model consists of field sources (mainly conductors and permanent magnets) and magnetic materials such as iron. Nonmagnetic elements, like air, are not
TABLE III. Number of 3D models of magnet pairs solved and approximated computation time for each of them, for one CPU.

\begin{tabular}{lcc}
\hline \hline Cross talks & Number of models & CPU time (h) \\
\hline QD2-DL high field & 25 & $2-2.5$ \\
QD3-DL low field & 16 & $2-2.5$ \\
QD3-DL low field & 15 & $2-2.5$ \\
QD3-SD1 & 106 & $2-3$ \\
QF4-SF2 & 180 & $2-3$ \\
QF6-DQ1 & 18 & 2 \\
QF8-DQ1 & 2 & 2 \\
\hline \hline
\end{tabular}

modeled, and the field at a given point is computed by summing up the contributions of all individual elements with nonzero current or magnetization. Solving a RADIA model consists of computing iteratively the magnetizations of all elements.

If a RADIA model includes two magnetically interacting magnets $\mathrm{A}$ and $\mathrm{B}$, the contribution of $\mathrm{A}$ at a given point is simply the sum of the fields produced by its individual elements. We used this fact in the next sections to separate the contributions from different magnets, which are easier to understand than the sum of fields from A and B.

For each magnet pair and currents settings, two models were computed: one with the two magnets at their nominal distance, and another one with the magnets separated by $1 \mathrm{~m}$ and with the same symmetries. Then, gradient errors were computed as

$$
\frac{\Delta G}{G}=\frac{G_{M, D}-G_{\mathrm{REF}}}{G_{\mathrm{REF}}},
$$

where $G_{M, D}$ is the gradient of a magnet (which could be the main quadrupole or the perturbing dipole, sextupole or octupole) at nominal distance, while $G_{\mathrm{REF}}$ is the quadrupole gradient computed at large distance.

All the computations presented here were performed with RADIA's Python interface [12]. An object-oriented Python module for building realistic accelerator magnets was developed and used for cross talk computations [13] (an example quadrupole to sextupole cross talk computation script is available online).

The computations were performed on the ESRF cluster. Pairs of magnets with different current settings were computed in parallel, each model on a single CPU. The job scheduling was done with the SLURM software [14]. Table III gives an idea of the number of models solved and of the computation time.

\section{B. Magnetic measurement instruments and methods}

Integrated magnetic field and integrated multipoles were measured with moving stretched-wire benches developed in house $[15,16]$. These benches consist of two sets of linear stages moving a wire stretched within the bore of the magnet under test. The measurement of the wire positions 
TABLE IV. Magnet settings for quadrupole to other magnet cross talk measurements. The cycling current was $110 \mathrm{~A}$ and the cross talk was measured at $I_{0}=85 \mathrm{~A}$ (see Fig. 3). All the field integral scans were performed by moving the two extremities of the wire on a synchronized circular trajectory, and measuring the voltage on the fly. The parameters of the measurement sequence are given in Table $\mathrm{V}$.

\begin{tabular}{ll}
\hline \hline Step & \multicolumn{1}{c}{ Description } \\
\hline 1 & Quadrupole at $I_{Q}=I_{0}$ \\
2 & Quadrupole at $I_{Q}=-I_{0}$ \\
3 & Quadrupole at $I_{Q}=I_{0}$, other magnet at $I_{M}=0 \mathrm{~A}$ \\
4 & Quadrupole at $I_{Q}=-I_{0}$, other magnet at $I_{M}=0 \mathrm{~A}$ \\
\hline \hline
\end{tabular}

and induced voltages yields the integral of the field along the wire. The integrated field multipoles are deduced from these measurements. Such a method is very convenient for measuring small magnet bores, as a wire can always be stretched through small apertures.

The deviations of the magnetic field induced by cross talks are small compared to the nominal fields. The field errors presented in the next sections are the differences between the integrated field multipoles with the two magnets interacting, and the integrated field multipoles of individual magnets.

Table IV describes the main steps of a magnetic measurement sequence of the cross talk between a quadrupole and another magnet. The parameters of the measurement sequence are given in Table V.

It should be noted that the field has to be measured in normal and reverse polarity in order to separate the effects from the two magnets. For practical reasons, the magnets are not aligned on the benches with the same accuracy as in the storage ring tunnel. On the benches, the magnetic axes of the two magnets can be misaligned by $1 \mathrm{~mm}$ transversely: this leads to feed-down terms which are easily

TABLE V. Measurement parameters. The bench control and analysis software is available online [16]. The wire is moved by two sets of Newport ILS100-CC and IMS100V stages driven by a Newport XPS motion controller. The induced voltage was measured with a Keithley $2182 \mathrm{~A}$ voltmeter.

\begin{tabular}{lcc}
\hline \hline Parameter & Value & Unit \\
\hline Wire trajectory & Circular & \\
Field component measured & Radial & \\
Measurement radius & $10-15$ & $\mathrm{~mm}$ \\
Wire velocity & 5 & $\mathrm{~mm} / \mathrm{s}$ \\
Integration time & $0.06-0.1$ & $\mathrm{~s}$ \\
Points per turn & 128 & \\
Turns & $2 \times 4$ & $\mathrm{~mm}$ \\
Wire length & 905 & $\mathrm{~mm}$ \\
Wire diameter & 0.1 & \\
Wire material & TiAl4V6 & $\mathrm{mm}$ \\
Wire sagitta & 0.013 & \\
\hline \hline
\end{tabular}

eliminated by bipolar measurements. To be more specific, the dipole field is affected by the quadrupole gradient feeddown in the dipole to quadrupole cross talk, and the gradient is affected by the sextupole strength in the quadrupole to sextupole case. This may occur even at zero sextupole current due to the remanent sextupole field and the large transverse misalignment of the magnet axes.

As already mentioned, the wire-based methods employed for measuring magnetic cross talks have no longitudinal resolution. The measured voltage varies with the integral of a component of the field along the wire: it can be understood as a projection of the field. Other alternative methods excite the wire with current: monitoring its deformation gives information on the longitudinal field distribution [17-21]. However, to our knowledge, these methods are not sensitive enough to accurately localize small field errors in the range of the percent of the nominal field.

Another possibility would have been to use a Hall probe bench, but the accurate positioning of a probe along the axes of small aperture magnets would have not been possible with the benches available at the ESRF. These benches are dedicated to planar undulator measurements and take advantage of the lateral openings of such devices.

\section{RESULTS}

\section{A. Permanent magnet dipoles and quadrupoles}

\section{Field}

The cross talk between permanent magnet (PM) dipoles and quadrupoles was simulated at the early stage of the project [22] and was measured on preseries magnets in

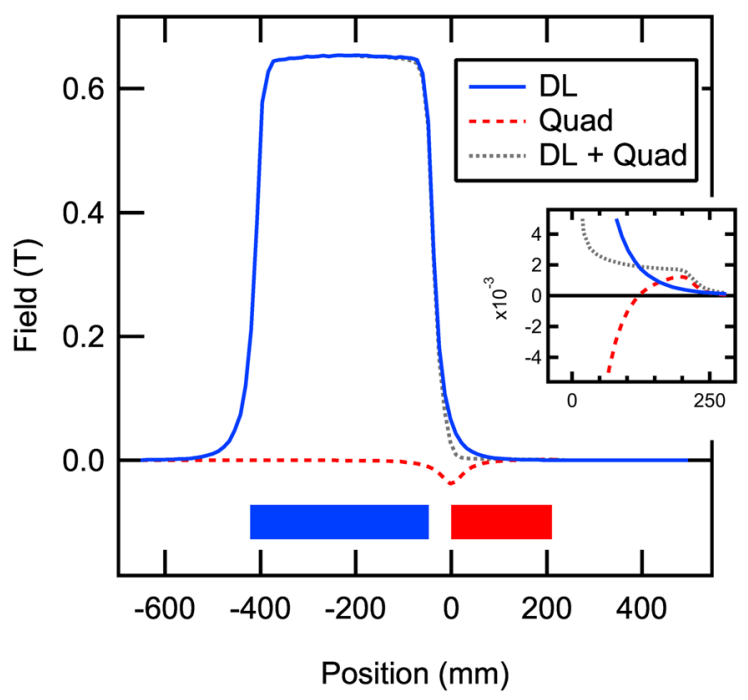

FIG. 4. Simulated longitudinal profile of the vertical field of interacting DL1 high field dipole module $(0.67 \mathrm{~T})$ and QD2 moderate gradient quadrupole $(57 \mathrm{~T} / \mathrm{m}, 94.5 \mathrm{~A})$. The small graph inserted on the right shows the field distribution inside the quadrupole. 


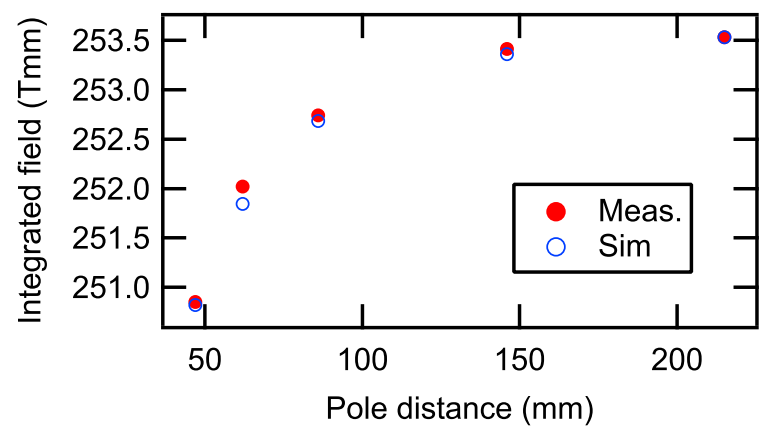

FIG. 5. Integrated field of a DL1 high field dipole and QD2 quadrupole assembly versus pole distance. Red disks (Meas): stretched-wire measurements; blue circles (Sim): simulations. An offset was added to make the measured and simulated field equal at a large distance. The distance errors were estimated to be $\pm 1 \mathrm{~mm}$ and the repeatability of the measurements was estimated to be $\pm 0.01 \%$.

2017. The simulations have shown that the cross talk induces a dipole field with opposite polarity localized at the quadrupole edge, as shown in Fig. 4. As expected, the sum of the fields from the dipole and quadrupole magnets decreases faster on the side where the magnets are interacting. It could also be noted that the sum of dipole and quadrupole fields results in a small dipole component propagating all along the quadrupole. When the magnets are positioned at $47 \mathrm{~mm}$ pole-to-pole distance, the overall integrated field is reduced by $1.08 \%$.

Figure 5 shows the simulated and measured integrated field (i.e. magnet angle) for a few values of pole-to-pole distance. The agreement between the two sets of points is very good. After subtracting an offset from the measurement, corresponding to the imperfect tuning of the dipole angle in laboratory, the relative error is $1.2 \times 10^{-4}$ at $47 \mathrm{~mm}$ pole distance (nominal) and reaches $6.9 \times 10^{-4}$ at $62 \mathrm{~mm}$ pole distance (worst case).

One may expect the cross talk to depend on the saturation of the magnets. Indeed, the reverse dipole field created by the quadrupole varies with its polarization. It results in an overall bending angle depending on the quadrupole current. This effect was measured for a permanent magnet dipole module positioned at nominal distance from a quadrupole; the results are shown in Fig. 6. The integrated field appears to be constant for quadrupole currents up to $70 \mathrm{~A}$, when the magnetic permeability of the quadrupole is high. At higher current, the quadrupole induced reverse field decreases as the quadrupole saturates, leading to a larger bending angle. This effect is almost linear for currents ranging from 80 to 110 A with a slope

$$
\frac{\Delta B_{\mathrm{INT}}}{B_{\mathrm{INT}} \Delta I} \approx 1.8 \times 10^{-4} \mathrm{~A}^{-1},
$$

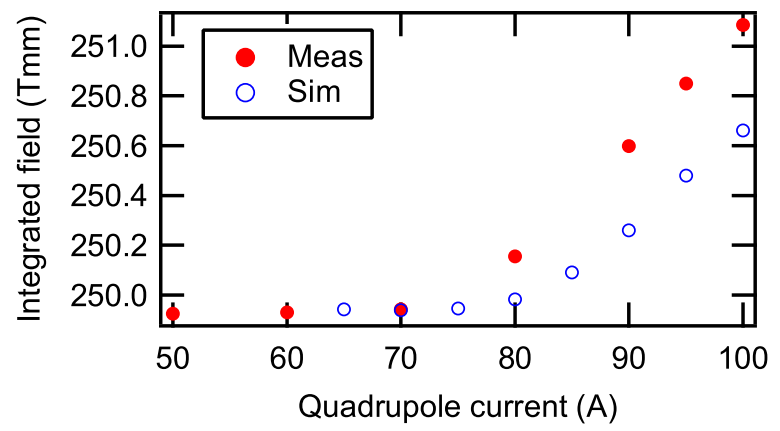

FIG. 6. Integrated field of a DL1 high field dipole and quadrupole assembly versus quadrupole current at $47 \pm 1 \mathrm{~mm}$ pole distance. An offset was added to make the data equal at low current. The repeatability of the measurements was estimated to be $\pm 0.01 \%$. The discrepancy between the measurement and the simulation comes probably from the magnetization curve used for the computations.

where $B_{\mathrm{INT}}$ is the integral of the field along the beam axis. This angle error is not negligible but can be canceled out by the dipole correctors installed all along the ring. It did not create any difficulty during the commissioning of the storage ring.

\section{Field gradient}

The effect of a neighboring dipole on the gradient of a quadrupole is similar to what was observed on the dipole field: a spurious gradient is observed at the dipole edge, as shown in Fig. 7. The main impact is the reduction of the integrated gradient by the shorter field decay on the dipole side, by $0.79 \%$ at the quadrupole current plot in the figure and $0.88 \%$ at nominal current.

The cross talks between permanent magnet dipoles and quadrupoles were integrated in the lattice model by adding

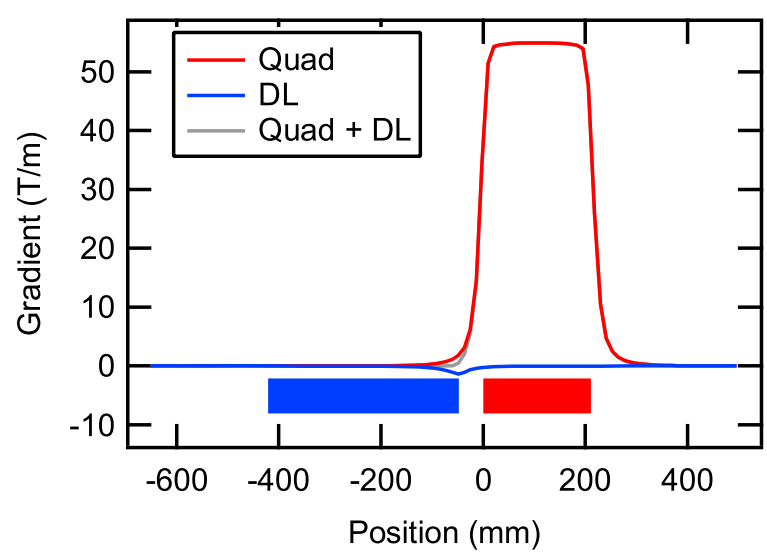

FIG. 7. Longitudinal gradient profile along a permanent magnet dipole (DL) and a moderate gradient quadrupole magnet. The cross talk induced gradient in the dipole can be modeled as a thin lens at the edge of the dipole, with opposite polarity. The quadrupole current was set to $94.5 \mathrm{~A}$ for this computation. 
a thin lens with opposite polarity at the dipole edge, and a reverse kick angle at the quadrupole edge. The lattice was then rematched to restore linear optics and maintain performance.

\section{B. Moderate gradient quadrupoles and sextupoles}

The quadrupole to sextupole cross talk has a similar effect on the gradient as the quadrupole to dipole cross talk: it acts as a thin lens with opposite polarities at the edge of the sextupole, shortening the magnetic length on the interacting side. The longitudinal distribution is shown in Fig. 8. The integrated field gradient is reduced by $0.30 \%$ due to the cross talk.

The sextupolar error induced by the quadrupole has a different profile: it acts as an opposite sextupole lens at the quadrupole edge and a sextupole component all along the quadrupole. The cross talk reduces the integrated sextupole strength by less than $0.1 \%$ and its impact on the strength was neglected in the lattice model.

The cross talk errors are bivariate functions: they depend both on the quadrupole and sextupole currents. Contour plots of the integrated gradient errors are given in Fig. 9. These plots show that, as the gradient error comes mostly from the sextupole, it is driven by its saturation. As the
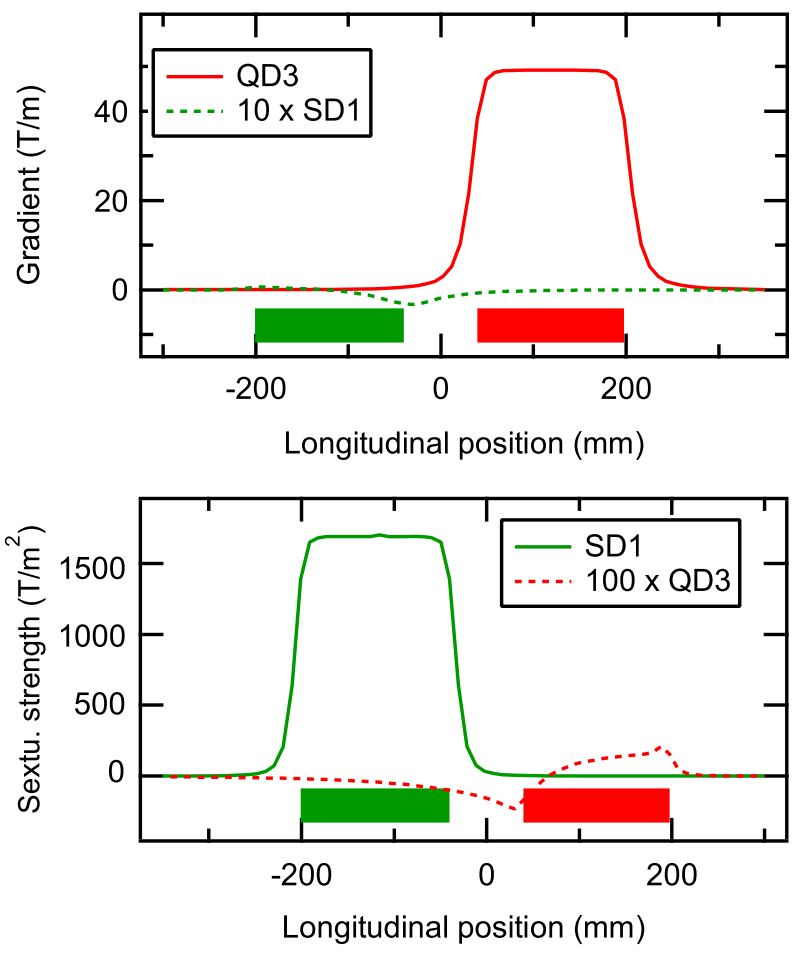

FIG. 8. Longitudinal distribution of the spurious gradient and sextupole strength $1 / 2 d^{2} B_{z} / d x^{2}$ for a SD1 sextupole (powered at $60 \mathrm{~A}$ ) placed at $75 \mathrm{~mm}$ from a QD3 quadrupole (powered at 80 A). Top: gradient from the quadrupole and gradient error from the sextupole (magnified by a factor of 10). Bottom: sextupole strength from the sextupole and error from the quadrupole (magnified by a factor of 100).
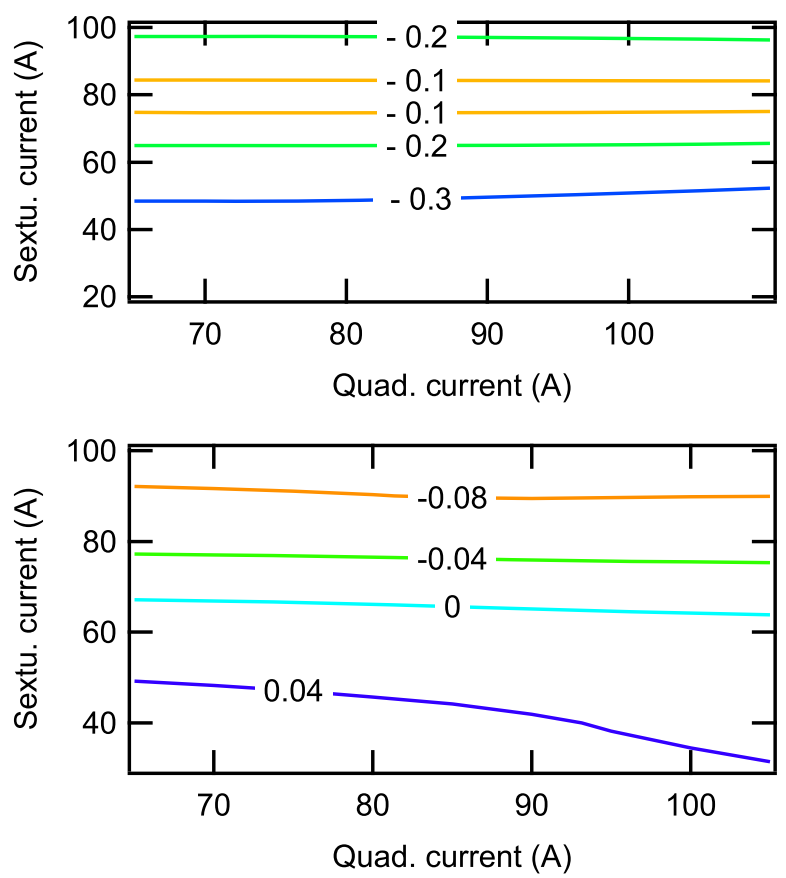

FIG. 9. 2D maps of the integrated gradient errors $\Delta G / G$ (in $\%$ of the QF4 integrated gradient). The contour plot on the top shows the contribution from the SF2 sextupole and the plot on the bottom shows the contribution from the QF4 quadrupole, at $75 \mathrm{~cm}$ pole-to-pole distance. The integrated gradient error is dominated by the response of the sextupole yoke to a quadrupole fringe field; the contribution of the quadrupole yoke is almost negligible. All these values were obtained from $3 \mathrm{D}$ simulations.

sextupole current increases, the permeability of its poles decreases and the inverse gradient at its edge goes down.

The integrated gradient error obtained from 3D simulations was compared to the value measured with a moving stretched wire. The results, given in Table VI, show a very good agreement between simulations and measurements.

\section{Moderate gradient quadrupoles and octupoles}

Based on what we have learned with dipole to quadrupole and sextupole to quadrupole cross talks, we would have expected to observe a localized gradient with opposite polarity at the octupole edge. This is not the case: the spurious gradient has the same polarity as the main quadrupole gradient, and is distributed all along the

TABLE VI. Measured and simulated value of the integrated gradient error, for a SF2 sextupole installed at $75 \pm 0.5 \mathrm{~mm}$ from a QF4 quadrupole.

\begin{tabular}{lcc}
\hline \hline Parameter & Value & Unit \\
\hline Quadrupole current & 85 & $\mathrm{~A}$ \\
Sextupole current & 0 & $\mathrm{~A}$ \\
$\Delta G / G$ (simulation) & -0.30 & $\%$ \\
$\Delta G / G$ (measurement) & -0.31 & $\%$ \\
\hline \hline
\end{tabular}




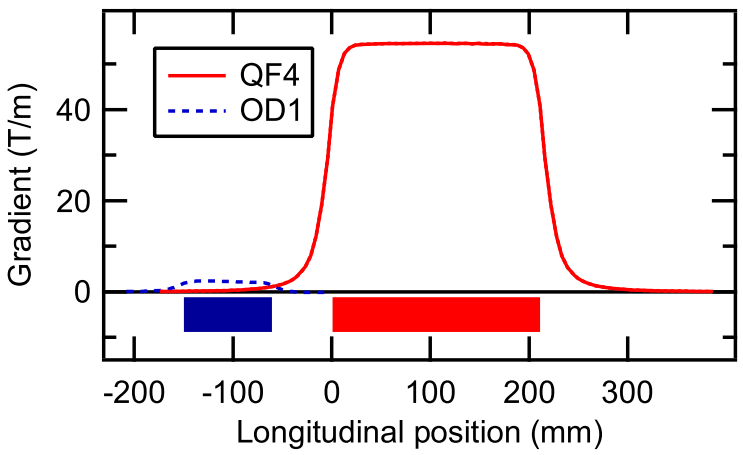

FIG. 10. Longitudinal distribution of the spurious gradient for a OD1 octupole (at $0 \mathrm{~A}$ ) placed at $60 \mathrm{~mm}$ from a QF4 quadrupole (powered at $90 \mathrm{~A}$ ).

magnet. Figure 10 shows the longitudinal distribution of the gradient errors.

It it also noticeable that the gradient errors induced by the cross talks with octupoles are by far the largest errors, reaching almost $+1.8 \%$ at low octupole and high quadrupole currents, as shown in Fig. 11.

Table VII gives the simulated and measured integrated gradient error, at two quadrupole currents. The simulations are in very good agreement with the measurements, with relative discrepancies in the range of $10^{-4}$.
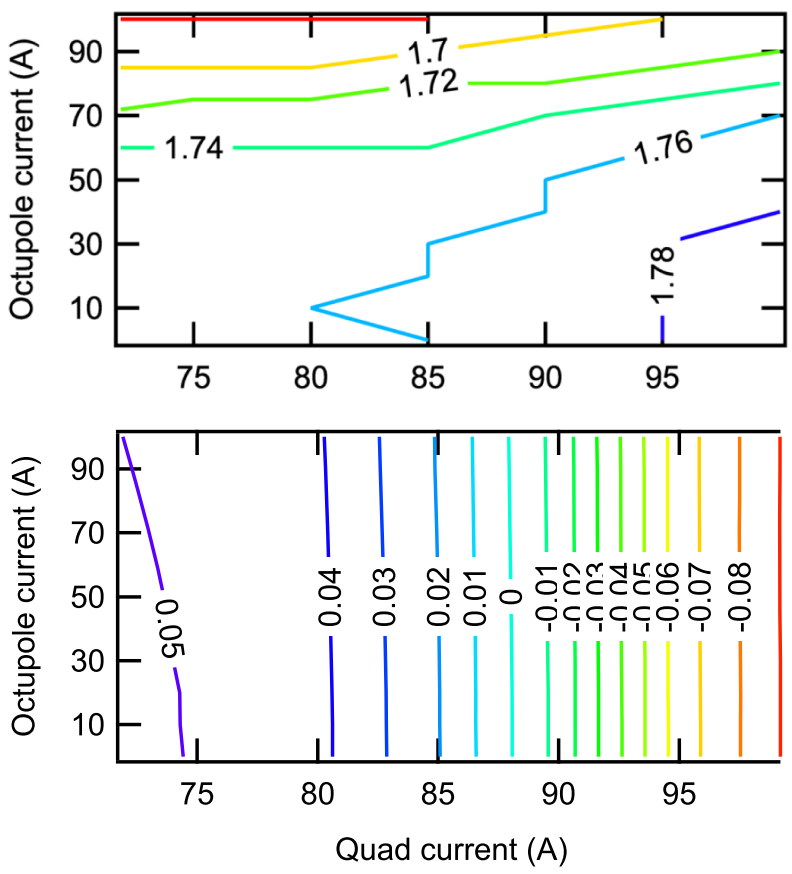

FIG. 11. 2D maps of the integrated gradient errors $\Delta G / G$ (in $\%$ of the QF4 integrated gradient), where the upper (respectively lower) plot shows the contribution of the OD1 (respectively $\mathrm{QF} 4)$, at $60 \mathrm{~cm}$ pole-to-pole distance. These two curves shows that the integrated gradient errors are mostly created by the reaction of the octupole yoke to a quadrupole fringe field: the errors induced by the quadrupole yoke are more than 1 order of magnitude lower. All these values were obtained from 3D simulations.
TABLE VII. Measured and simulated value of the integrated gradient error, for a OD1 octupole installed at $60 \pm 0.5 \mathrm{~mm}$ from a QF4 quadrupole.

\begin{tabular}{lcc}
\hline \hline Parameter & Value & Unit \\
\hline Quadrupole current & 85 & $\mathrm{~A}$ \\
Octupole current & 0 & $\mathrm{~A}$ \\
$\Delta G / G$ (simulation) & 1.78 & $\%$ \\
$\Delta G / G$ (measurement) & 1.77 & $\%$ \\
Quadrupole current & 100 & $\mathrm{~A}$ \\
Octupole current & 0 & $\mathrm{~A}$ \\
$\Delta G / G$ (simulation) & 1.79 & $\%$ \\
$\Delta G / G$ (measurement) & 1.76 & $\%$ \\
\hline \hline
\end{tabular}

TABLE VIII. Simulated value of the integrated gradient error, for a DQ1 dipole-quadrupole installed at $90 \mathrm{~mm}$ from QF6 and QF8 high gradient quadrupoles.

\begin{tabular}{lcc}
\hline \hline Parameter & Value & Unit \\
\hline Quadrupole current & 90 & $\mathrm{~A}$ \\
DQ current & 85.5 & $\mathrm{~A}$ \\
$\Delta G / G$ QF6 & -0.08 & $\%$ \\
$\Delta G / G$ DQ1 & 0.04 & $\%$ \\
$\Delta G / G$ QF8 & 0.04 & $\%$ \\
$\Delta G / G$ DQ1 & -0.06 & $\%$ \\
\hline \hline
\end{tabular}

\section{High gradient quadrupoles and dipole quadrupoles}

As mentioned in the Introduction, the high gradient quadrupoles are saturated (Fig. 3). Saturated iron poles mean more magnetic flux outside the magnet and more fringe field: one would have expected these magnets to be severely affected by magnetic cross talks. Simulations indicated this is not the case, as shown in Table VIII. We identified two reasons for these moderate cross talks: the increased distance between magnets at the location of high gradient quadrupoles (i.e., at the center of the arcs), and the reduced magnet apertures that allow higher gradients to be reached.

\section{INTEGRATION OF THE MAGNETIC CROSS TALKS IN THE LATTICE MODEL}

The hybrid multibend achromat lattice [1] lifetime, dynamic aperture and emittance performance strongly rely on how the linear optics are controlled. Localized field errors of the order of one percent can substantially degrade these parameters and are difficult to correct using global optics correction as is generally the case in storage ring light sources. It is therefore essential to correct all known errors at the source by either adapting the magnet calibration curves or integrating them in the lattice model. At ESRF, the latter option was adopted to account for the magnetic cross talks. Only gradient errors were considered, by adding a thin magnetic lens at the entrance or exit of the affected magnets in order to be as close as possible to the 
modeled longitudinal field distribution. The only exceptions are the octupole for which the gradient error is applied through the full magnet length. Sextupole field errors, considered as second order effects, were not integrated in the lattice model. Beam experiments are nevertheless planned in the near future to assess their impact on operation.

Figure 12 shows the optics distortions introduced by the magnetic cross talks, in blue, and the residual errors after correction, in red, for one cell of the HMBA lattice. The gradient errors introduced by the cross talks induced a $\beta$ beating as large as $30 \%$ and a dispersion beating of several millimeters. These are of course not acceptable as the design $\beta$ beating is of the order of a few percent and the design dispersion beating approximately $1 \mathrm{~mm}$. However, once the magnetic errors were introduced in the model in order to be as close as possible to reality, it was possible to rematch the lattice and restore linear optics properties. The residuals, shown in red in Fig. 12, are considered negligible.
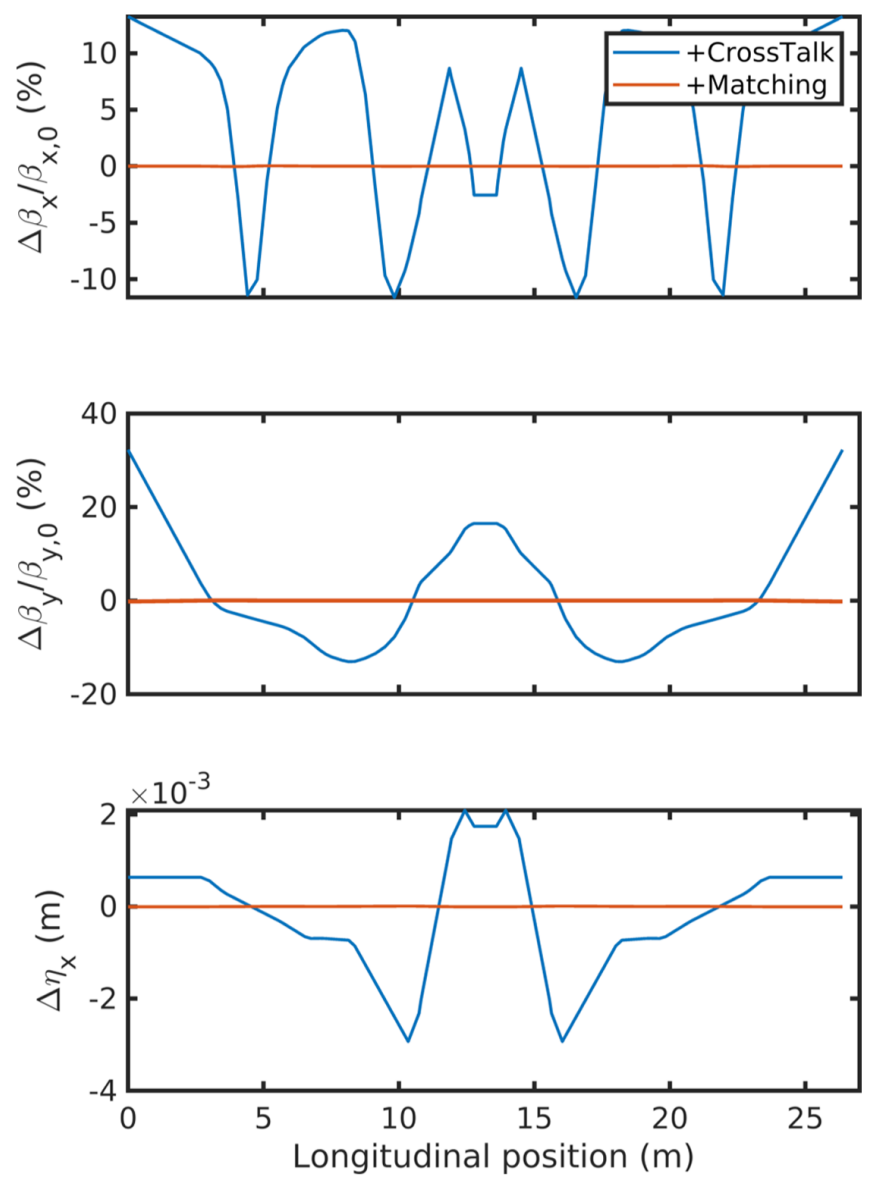

FIG. 12. Optics distortions introduced by magnetic cross talks before and after correction (matching) for one HMBA cell. The top figure is the horizontal $\beta$ function, the middle one the vertical, and the bottom one the dispersion. The comparison is done with respect to the design lattice.
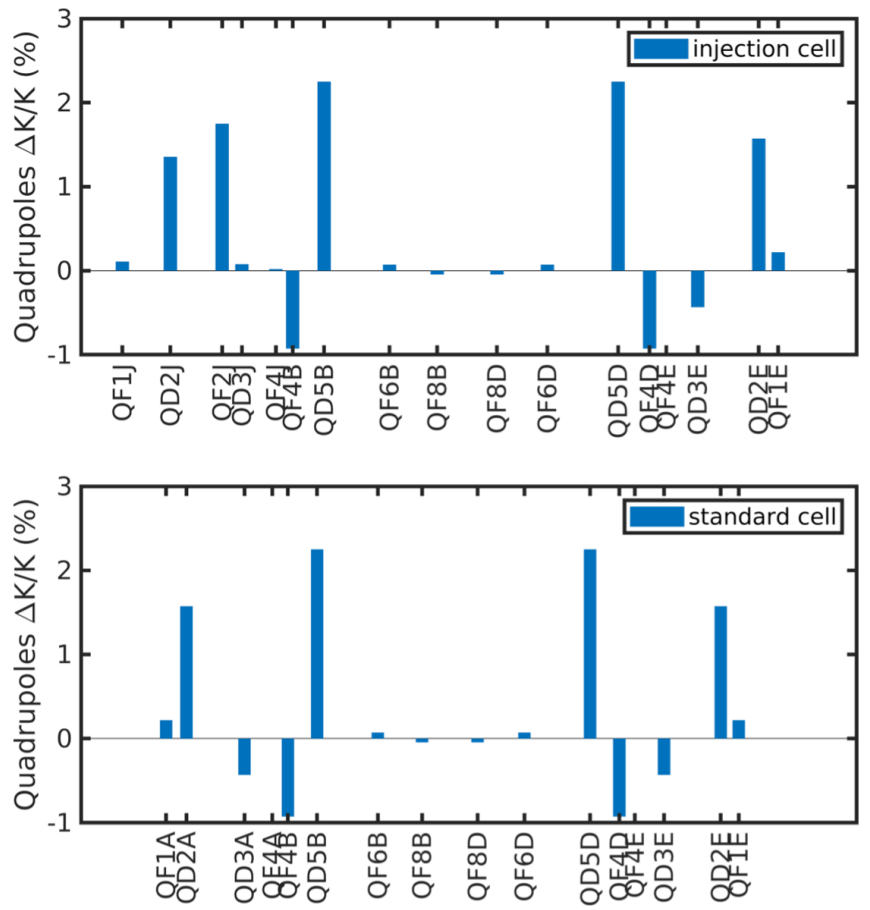

FIG. 13. Gradient corrections required to restore the design linear optics.

Figure 13 shows the gradient corrections that were applied to restore the design linear optics for the standard and injection HMBA cells. As expected, most of the corrections are concentrated on the moderate gradient quadrupoles where the magnetic cross talks are the strongest.

Even though they are now well integrated in the lattice and the ESRF-EBS storage ring successfully resumed user service mode (USM) operation and delivers design performance in most aspects [23,24], the initial commissioning phase of the machine was performed with these strong field errors uncorrected. While clear difficulties were faced when trying to inject and store beam, potentially indicating very low dynamic aperture, these were associated with several unrelated issues to which the cross talk induced magnetic field errors contributed only partially. However, the betatron tune represents a simple measurable quantity that was available from almost the first injections to determine how well the lattice is modeled and directly relates to the gradient errors.

Table IX summarizes the model and measured tunes for various operating and lattice design scenarios. The model predicts tune shifts associated with the magnetic cross talks of approximately 0.5 and 1.3 units in the horizontal and vertical planes respectively, which are fully restored after correction. These values are confirmed in the vertical plane, with measurements giving a tune shift of approximately 1.4 with respect to the design model, but not in the horizontal plane where the measurements give -0.3 instead of 0.5 . These measurements, taken during the initial phase of the 
TABLE IX. Simulated and measured tunes for various lattice iterations.

\begin{tabular}{lcc}
\hline \hline & $Q_{h}$ & $Q_{v}$ \\
\hline Design values & 76.21 & 27.34 \\
Design values + cross talks & 76.70 & 26.00 \\
Corrected model & 76.21 & 27.34 \\
Measured values + cross talks & 75.90 & 25.90 \\
Measured values + corrections & 76.21 & 26.24 \\
\hline \hline
\end{tabular}

commissioning when the cross talk were not integrated, are based on the first turns trajectory data and may suffer significant systematic errors. Nevertheless, the ESRF-EBS storage ring optics errors after corrections and optimization are estimated to be approximately $1.5 \%$ beta beating in both planes and $1 \mathrm{~mm}$ rms dispersion error. This level is in agreement with model expectation and compatible with USM operation. It was obtained with lower than expected correction strengths and therefore confirms the excellent magnetic modeling of the machine. The remaining residual errors are expected and relate mostly to the nonzero closed orbit and the resulting feed-down effects from the sextupoles that cannot be perfectly corrected using nearby quadrupoles.

\section{CONCLUSION}

The magnetic cross talks between neighboring magnets of the ESRF-EBS storage ring are not negligible. They affect mainly the dipoles' bending angles and the calibration of the quadrupoles. The dipoles are permanent magnets without trimming coils: their bending angles were tuned in the laboratory taking into account angle errors due to the cross talks. The calibration of the $50 \mathrm{~T} / \mathrm{m}$ quadrupoles is affected by neighboring dipoles, sextupoles and octupoles by up to $1.8 \%$. The cross talks are magnet dependent: dipoles and sextupoles placed close to quadrupoles create opposite gradient at the edges, while octupoles introduce a gradient with the same polarity all along the magnet.

Rather surprisingly, the strongly saturated $90 \mathrm{~T} / \mathrm{m}$ quadrupoles are weakly impacted by the cross talks. This is probably due to their smaller aperture (i.e., shorter fringe field extension) and to the increased distances between magnets at their locations. Simulations at various currents have shown that the cross talk induced gradient errors are relatively independent of the saturation of quadrupole magnets.

The quadrupoles do affect the strengths of neighboring sextupole and octupole magnets by a small amount (less than $0.1 \%$ for the sextupole). This did not cause any additional issues.

The magnetic cross talks made the commissioning more difficult, as they introduced significant focusing strength errors on several quadrupole families. The correct strengths were obtained from 3D magnetic simulations run in a short time during the ESRF-EBS early commissioning. The gradient errors were integrated in the lattice model by modifying the main quadrupole strengths and by adding thin lenses with opposite polarities (dipoles, sextupoles) or thick lenses with the same polarity (octupoles).

Some of the simulation results were compared to stretched-wire magnetic measurements. The agreement between simulated and measured values is very good: relative errors are in the $10^{-4}$ range. This makes us feel confident in the simulated longitudinal error profiles which were not measurable with our measurement benches.

It is difficult to separate the impact of cross talk corrections from the effect of other commissioning activities, such as improvement of the steering, beam based alignment and correction of individual magnet calibration errors: all these improvements were done at the same time.

Finally, the design lattice parameters were reached rapidly, demonstrating that the machine performance is not affected by the magnetic cross talks after their integration in the model. Most light source upgrade projects are based on compact lattices which are potentially sensitive to cross talks. These cross talks do not appear to be an issue if they are correctly characterized, which implies a significant effort in magnetic computations and measurements.

[1] L. Farvacque, N. Carmignani, J. Chavanne, A. Franchi, G. Le Bec, S. Liuzzo, B. Nash, T. Perron, and P. Raimondi, A low-emittance lattice for the ESRF, in Proceedings of the 4th International Particle Accelerator Conference, IPAC2013, Shanghai, China, 2013 (JACoW, Shanghai, China, 2013), pp. 79-81.

[2] J. A. Eriksson, A. Andersson, M. Johansson, D. Kumbaro, S. C. Leemann, C. Lenngren, P. Lilja, F. Lindau, L.-J. Lindgren, L. Malgren, J. Moder, R. Nilsson, M. Sjstrm, J. Tagger, P. F. Tavares, S. Thorin, E. Walln, S. Werin, B. Anderberg, and L. Dallin, The MAX IV synchrotron light source, in Proceedings of the 2nd International Particle Accelerator Conference, IPAC11, San Sebastián, Spain (EPS-AG, Spain, 2011), pp. 3026-3028.

[3] M. Borland, V. Sajaev, Y. Sun, and A. Xiao, Hybrid sevenbend-achromat lattice for the advanced photon source upgrade, in Proceedings of the 6th International Particle Accelerator Conference, IPAC15, Richmond, VA, USA (JACoW, Richmond, VA, USA, 2015), pp. 1776-1779.

[4] H. Tanaka, T. Ishikawa, S. Goto, S. Takano, T. Watanabe, and M. Yabashi, Spring-8 upgrade project, in Proceedings of the 7th International Particle Accelerator Conference, IPAC16, Busan, Korea (JACoW, Busan, Koera, 2016), pp. 2867-2870.

[5] C. Steier, J. Byrd, H. Nishimura, D. Robin, S. De Santis, F. Sannibale, C. Sun, M. Venturini, and W. Wan, Physics design progress towards a diffraction limited upgrade of the ALS, in Proceedings of the 7th International Particle Accelerator Conference, IPAC16, Busan, Korea (JACoW, Busan, Koera, 2016), pp. 2956-2958. 
[6] E. Karantzoulis, The diffraction limited light source ELETTRA 2.0, in Proceedings of the 8th International Particle Accelerator Conference, IPAC17, Copenhagen, Denmark (JACoW, Copenhagen, Denmark, 2017), pp. 2660-2662.

[7] G. Le Bec, J. Chavanne, C. Benabderrahmane, L. Farvacque, L. Goirand, S. Liuzzo, P. Raimondi, and F. Villar, High gradient quadrupoles for low emittance storage rings, Phys. Rev. Accel. Beams 19, 052401 (2016).

[8] M. Abliz, M. Jaski, A. Jain, M. Borland, G. Decker, and J. Kerby, Magnetic crosstalk simulation between Q2 and Lbend magnets of APS-U, Nucl. Instrum. Methods Phys. Res., Sect. A 913, 48 (2019).

[9] G. Le Bec, J. Chavanne, F. Villar, C. Benabderrahmane, S. Liuzzo, J.-F. Bouteille, L. Goirand, L. Farvacque, J.-C. Biasci, and P. Raimondi, Magnets for the ESRF diffraction limited light source project, IEEE Trans. Appl. Supercond. 26, 1 (2016).

[10] P. Elleaume, O. Chubar, and J. Chavanne, Computing 3D magnetic fields from insertion devices, in Proceedings of the Particle Accelerator Conference, Vancouver, BC, Canada, 1997 (IEEE, New York, 1997), pp. 3509-3511.

[11] O. Chubar, P. Elleaume, and J. Chavanne, A three-dimensional magnetostatics computer code for insertion devices, J. Synchrotron Radiat. 5, 481 (1998).

[12] O. Chubar, RADIA, https://github.com/ochubar/Radia.

[13] G. Le Bec, RADIA multipole magnets, https://gitlab.esrf.fr/ IDM/radia/radiamm.

[14] SLURM, https://slurm.schedmd.com/.

[15] G. Le Bec, J. Chavanne, and C. Penel, Stretched wire measurement of multipole accelerator magnets, Phys. Rev. ST Accel. Beams 15, 022401 (2012).
[16] G. Le Bec, Stretched wire lab, https://gitlab.esrf.fr/IDM/ swlab (2020).

[17] R. W. Warren and C. J. Elliott, New system for wiggler fabrication and testing, in Conference on Undulator Magnets for Synchrotron radiation and Free Electron Lasers, Trieste, Italy (1987).

[18] R. Warren, Limitation on the use of the pulsed-wire field measuring technique, Nucl. Instrum. Methods Phys. Res., Sect. A 272, 257 (1988).

[19] A. Temnykh, Vibrating wire field-measuring technique, Nucl. Instrum. Methods Phys. Res., Sect. A 399, 185 (1997).

[20] Z. Wolf, A vibrating wire system for quadrupole fiducialization, SLAC Report No. LCLS-TN-05-11, 2005.

[21] D. Arbelaez, T. Wilks, A. Madur, S. Prestemon, S. Marks, and R. Schlueter, A dispersion and pulse width correction algorithm for the pulsed wire method, Nucl. Instrum. Methods Phys. Res., Sect. A 716, 62 (2013).

[22] G. L. Bec, Magnet design at the ESRF, in 3rd Low Emittance Ring Workshop, Oxford, UK (2013), https:// indico.cern.ch/event/247069/.

[23] S. White, N. Carmignani, L. Carver, J. Chavanne, L. Farvacque, L. Hardy, J. Jacob, G. Le Bec, S. M. Liuzzo, T. Perron, Q. Qin, P. Raimondi, J.-L. Revol, and K. B. Scheidt, Commissioning and restart of ESRF-EBS, in Proceedings of the 12th International Particle Accelerator Conference, IPAC21, Campinas, SP, Brazil (JACoW, Campinas, SP, Brazil, 2021).

[24] P. Raimondi, N. Carmignani, L. R. Carver, J. Chavanne, L. Farvacque, G. Le Bec, S. M. Liuzzo, T. Perron, and S. White, The hybrid multibend achromat lattice commissioning at the European Synchrotron Radiation Facility (to be published). 